\title{
EFFECT OF TEMPERATURE VARIATION ON FLUID FLOW ACROSS A Cu-MICA MICROCHANNEL
}

\author{
Harneet KourKhajuria ${ }^{1}$, Simranjit Kaur ${ }^{2}$, Parveen Lehana ${ }^{3}$ \\ ${ }^{1}$ M.tech student, ${ }^{2}$ Assistant Professor, Dept. of E\&C, SSCET, Badhani, Pathankot, Punjab, India \\ ${ }^{3}$ Associate Professor, Dept. of Physics \& Electronics, University of Jammu, Jammu, India \\ harneet.destiny@gmail.com,simran2013@yahoo.com,klehanajournals@gmail.com
}

\begin{abstract}
Microfluidics is a science of fluid mechanics involving micro-scale dimensions. The research in miniaturization of microfluidic devices and its use for microfluidic applications is increasing exponentially. The success of microfluidics owes to its inherent potential of influencing other fields such as chemical synthesis, biological analysis, optics, and information technology, etc. Various factors may affect the microfluidic behavior such as nature of microfluids, surface tension, contact angle, channel shape, temperature, viscosity, etc. The objective of this paper is to investigate the effect of temperature on microfluidic flow across a Cu-Mica microchannel. The microchannel was fabricated using a screen printing technique followed by substrate etching. Three different microfluids namely Ethanol, Methanol, and Chloroform were considered for investigations. The angle of elevation of the microchannel was maintained constant during all the investigations. The analysis of results showed that the flow rate of microfluids is temperature and fluid dependent.
\end{abstract}

Keywords: Cu-Mica Microchannel, Microfluid, Microfluidics, Microfludic Flow.

***

\section{INTRODUCTION}

Microfluidics is considered as an interdisciplinary field in its adolescence. The success of microfluidics owes to its inherent potential of influencing other fields such as chemical synthesis, biological analysis, optics, and information technology, etc. Microfluidics is defined technically as science of fluid mechanics studied at micro scale [1]. It is formed by integration of many parental fields such as molecular biology, molecular analysis, bio defense, and microelectronics and thus microfluidics has become the only solution to overcome the problems associated with development of high resolution and highly sensitive analytical methods used in microanalysis purposes $[1,2]$.

Microfluidics deals with the study of the behavior, precise control, and the manipulation of fluids constrained to submillimeter scale that is at micro scale [1]. When different micro-components capable of processing are integrated together with precisely manipulated small volumes of fluids preferably varying in range from pico-liter to micro- liter; a fluidic system is formed at micro scale called microfluidic system [1-3]. Microfluidic systems possess the ability to carry out high resolution and sensitive separations and detections using very small quantities of samples and reagents which make this system inexpensive requiring short time analysis [1]. Microfluidic devices essentially contain one or more microchannels of dimensions not greater than $1 \mathrm{~mm}$ to perform fluid analysis at micro domains [1, 2]. Most of microscale investigations of fluids are carried out using flow injection analysis as discussed in the succeeding section.

\subsection{Flow Injection Analysis}

Flow injection analysis (FIA) is a continuous flow technique used for chemical analysis using syringes. It provides a precise and good analytical performance with high reproducibility and sensitivity. Faster and high throughput analysis can be obtained at low costs using FIA techniques. Microfluidic devices are more advantageous than simple fluidic analysis since they require very small amount of reagents and chemicals which makes the analysis scalable to faster and high throughput oriented analysis [1-3].

The paper is divided into various sections. Dynamics of fluidic flow includes introduction to various parameters essential for microfluidic analysis such as surface tension, contact angle, and viscosity as discussed in Section 2. Section 3 describes the methodology involved for proposed investigations followed by results and discussions in Section 4 . Conclusions are drawn in Section 5.

\section{DYNAMICS OF FLUIDIC FLOW}

The important factors affecting dynamics of microfluidic flow are surface tension, contact angle, and viscosity. These are described as follows. 


\subsection{Surface Tension}

It is a contractive tendency of a liquid which allows it to resist an external acting force. Since the behavior of the liquid depends on cohesion forces acting between similar molecules, it has the dimension of force per unit length or energy per unit area $[4,5]$. The capillary action achieved is termed as perfect capillary action theoretically, when the gas pressure of fluid nearly equals the atmospheric pressure acting on the open end of channel $[4,5]$. The increase in capillary forces with time depends on channel aspect ratio. Higher the aspect ratio of the micro channel, faster is the displacement and this leads to increase of capillary forces with time [5]. The surface tension is determined by equation $[4,6]$ as follows:

$$
\gamma=\frac{U}{2 \delta^{2}}
$$

Where $\gamma$ is surface tension $(\mathrm{N} / \mathrm{m}), u$ is average cohesive energy of a molecule, $\delta$ is characteristic dimension of a molecule and $\delta^{2}$ represents the effective surface area of molecule.

Total energy $(E)$ stored in the interface is [6] given as

$$
E=\gamma S
$$

Where $S$ is total surface area of the interface

\subsection{Contact Angle}

Contact angle is an important parameter in slug formation process and wall adhesion equations. It is used to determine the shape of the fluid interface. It is calculated using Young's modulus law [7]. The capillary flow requires the maintenance of surface tension equilibrium which further can be achieved by varying the contact angle. The contact angle at a point of intersection of three interfaces is obtained by balancing the surface tensions at each interface. The range of contact angle is used to classify surfaces as wetting (hydrophilic) and nonwetting (hydrophobic). The wetting surfaces include the contact angle range as $0 \leq \theta<90^{\circ}$ where as the range of $90^{\circ} \leq \theta \leq 180^{\circ}$ refers to non-wetting surfaces $[6,7]$. The contact angle is given as.

$$
\gamma_{L G} \operatorname{COS} \theta=\gamma_{S G}-\gamma_{S L}
$$

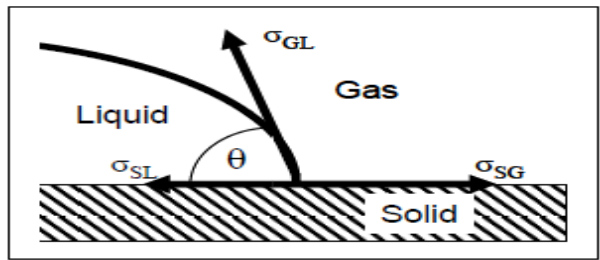

Fig.-1: Young's contact angle configuration [7].
The interaction of gas and liquid phases with the channel wall can be determined by specifying the contact angle which also affects their shape, size as well as velocity [7, 8]. There are different factors that can influence a contact angle. These factors are surface topography, liquid purity, equilibration time, temperature, surface impurities, sessile drop size as well as thermodynamic stabilities [6, 7, 8]. The effect of temperature changes on contact angle holds an important consideration in microfluidic analysis. It is a deterministic factor to acquire desired précised results of applications for which the microfluidic system is designed. However in case of liquids, surface tension of liquids is observed to be more sensitive to effects of temperature changes [9]. Sessile drop size is another factor that affect contact angle [9]. Capillary length is a parameter that determines the maximum size of the droplet allowed to flow across a microchannel and is given as.

$$
\frac{1}{K}=\left(\frac{\gamma_{L}}{\rho_{L g}}\right)^{\frac{1}{2}}
$$

Where $L$ is density of liquid and $1 / k$ is the capillary length [10].

\subsection{Viscosity}

The property of fluid which poses a friction or internal resistance to the flow of fluid is termed as the viscosity. The fluids with low viscosity are faster flowing as compared to highly viscous fluids. The choice of microfluids for proposed investigations owes to their properties and applications in various fields such as bio-medicinal research [11, 12, 13], bio-

\begin{tabular}{|c|c|c|c|c|c|}
\hline SNo. & Characteristic & & Ethanol & Methanol & Chloroform \\
\hline 1. & Colour & & Colourless & Colourless & Colourless \\
\hline 2. & Odour/aste & & $\begin{array}{l}\text { Sweet smelling in dilute } \\
\text { sols and burning taste } \\
\text { in concentrated sols. }\end{array}$ & Distinct borning taste & Sweet smelling \\
\hline 3. & Volatile & & Flammable & Highly flammable & Not readily flammable \\
\hline 4. & $\begin{array}{l}\text { Physical } \\
\text { properties }\end{array}$ & $\begin{array}{l}\text { MP } \\
\text { B.P } \\
\text { Viscosity } \\
\text { Density }\end{array}$ & $\begin{array}{l}-114.1^{\circ} \mathrm{C} \\
78.5^{\circ} \mathrm{C} \\
0.001095 \mathrm{Nsm}^{2} \\
0.789 \mathrm{~g} \mathrm{~cm}^{2}\end{array}$ & $\begin{array}{l}-97 . .^{\circ} \mathrm{C} \\
64.7^{\circ} \mathrm{C} \\
0.00056 \mathrm{Ns} \mathrm{m}^{2} \\
0.7918 \mathrm{~g}^{2} \mathrm{~cm}^{2}\end{array}$ & $\begin{array}{l}-63.5^{\circ} \mathrm{C} \\
61.7^{\circ} \mathrm{C} \\
0.00053 \mathrm{Nsm}^{2} \\
1.4832 \mathrm{~g} \mathrm{~cm}^{-2}\end{array}$ \\
\hline 5. & Applications & & 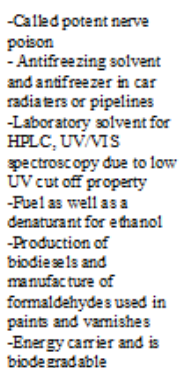 & 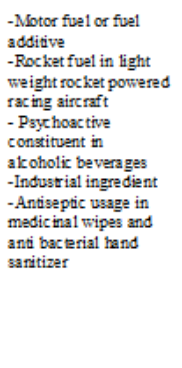 & $\begin{array}{l}\text {-Called molecular life } \\
\text { save } \\
\text {-Anesthe tic a agnt in } \\
\text { painful operations } \\
\text {-Production of } \\
\text { chlorodifluoromethane, } \\
\text { dyes, pesticides } \\
\text {-Laboratory solvent in } \\
\text { pharmaceutical } \\
\text { industries } \\
\text {-Reagent in or ganic } \\
\text { synthesis } \\
\text {-Working fluid in heat } \\
\text { engines }\end{array}$ \\
\hline
\end{tabular}
detections $[14,15,16]$ and industrial applications [12, 16, 17]. A few of these characteristics are described in Table1.

Table.-1: Fluid Characteristics. 


\section{METHODOLOGY}

The methodology for proposed investigations to study effect of temperature on fluid flow across a $\mathrm{Cu}$-Mica microchannel is divided into three stages: $\mathrm{Cu}-\mathrm{Mica}$ microchannel fabrication, pattern etching, and temporal FIA.

\section{Stage 1: Cu-Mica microchannel fabrication}

A $\mathrm{Cu}$-Mica microchannel of channel length $3.6 \mathrm{~cm}$ is fabricated using screen printing fabrication method. The shape of this $\mathrm{Cu}$-Mica microchannel is chosen as ' $\mathrm{Y}$ ' shaped designed using coral draw and fabricated on Mica base with Copper as boundaries. The channel width is fixed as $1 \mathrm{~mm}$. The microchannel pattern is transferred to $\mathrm{Cu}$-Mica board.

\section{Stage 2: Pattern etching}

The microchannel patterned substrate is subjected to etching in which unwanted Copper from substrate is removed from unexposed areas leaving behind the $\mathrm{Cu}$ microchannel on Mica base. The etchant used is Ferric Chloride solution prepared in the ratio of 3:1 with water in a plastic tray stirring continuously using a glass rod. The complete etching took 1215 minutes. After the pattern is etched completely the substrate is washed under running water and then swab coating of substrate using acetone was done to ensure the dirt free channel fabrication for FIA. The etched pattern used for FIA to carry out proposed investigations is as shown in Fig. 2.

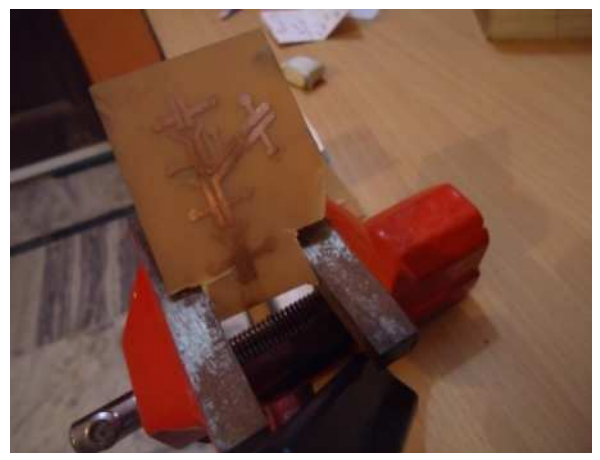

Fig.-2: Etched Cu-mica microchannel.

\section{Stage 3: Temporal FIA}

The proposed investigations were accomplished using three fluids- Ethanol, Methanol, and Chloroform. These fluids are taken in three metal syringes separately and then made to flow across a $\mathrm{Cu}-\mathrm{Mica}$ microchannel fixed at 450 angle of elevation and flow injection analysis is made by raising the temperature from room temperature to two different temperatures 400 and 500. The flow of these fluids at different temperature and fixed elevation angle of channel is observed and recorded using a digital movie camera. The temperature of micro channel is raised using an IR lamp set up as shown in
Fig. 3. For convenience of representation, the unit second represents centisecond in all the measurements in this paper.

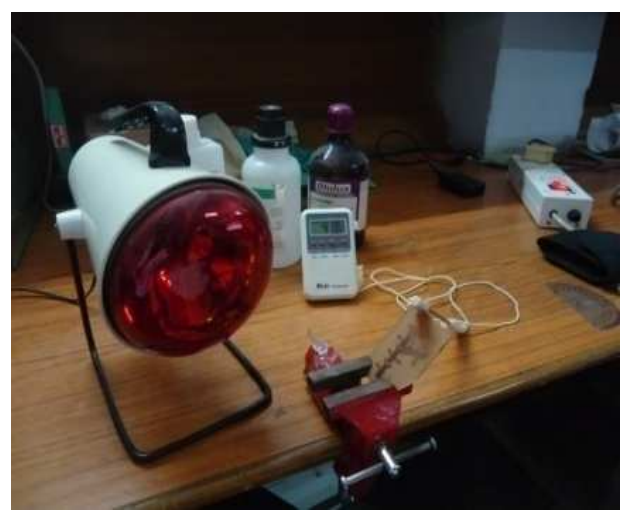

Fig.-3: IR set up

\section{RESULTS AND DISCUSSIONS}

The results obtained from the analysis of video clips of the microfluids flow in the $\mathrm{Cu}-\mathrm{Mica}$ microchannel are listed in Table II and plotted in Fig. 4 and Fig. 5. Flow time of fluids at different temperatures (Fig. 4) shows that flow time of chloroform is less as compared to Ethanol and Methanol. Ethanol takes comparatively more time to flow across the microchannel even at higher temperature. At higher temperature Chloroform flow is more streamline and that of Ethanol is irregular.

At room temperature all the fluids - Ethanol, Methanol, and Chloroform flow in a laminar way, i.e., stream lined flow is observed. Flow rate varies with the fluid used, Chloroform is the fastest fluid followed by Methanol, and then Ethanol in terms of speed of the flow.

At higher temperatures the flow pattern is irregular in case of Methanol and Ethanol but the flow pattern of Chloroform remains stream line or laminar. The speed of all fluids is higher as compared to the flow rate observed at room temperature.

Table 2 Effect of Temperature Change on Fluid Flow

\begin{tabular}{|l|l|l|l|l|l|l|}
\hline Microfluid & \multicolumn{3}{|c|}{$\begin{array}{c}\text { Time (s) } \\
\text { for Temperature }\end{array}$} & \multicolumn{3}{c|}{$\begin{array}{c}\text { Speed (cm/s) } \\
\text { for Temperature }\end{array}$} \\
\hline & $30^{\circ} \mathrm{C}$ & $40^{\circ} \mathrm{C}$ & $50^{\circ} \mathrm{C}$ & $30^{\circ} \mathrm{C}$ & $40^{\circ} \mathrm{C}$ & $50^{\circ} \mathrm{C}$ \\
\hline Ethanol & 0.07 & 0.05 & 0.04 & 51.43 & 72.00 & 90.00 \\
& 0 & 0 & 0 & & & \\
\hline Methanol & 0.04 & 0.03 & 0.02 & 90.00 & 120.0 & $\begin{array}{l}180.0 \\
0\end{array}$ \\
& 0 & 0 & 0 & & 0 & 0 \\
\hline $\begin{array}{l}\text { Chlorofor } \\
\mathrm{m}\end{array}$ & 0.02 & 0.02 & 0.01 & 144.0 & $\begin{array}{l}180.0 \\
0\end{array}$ & $\begin{array}{l}360.0 \\
0\end{array}$ \\
\hline
\end{tabular}




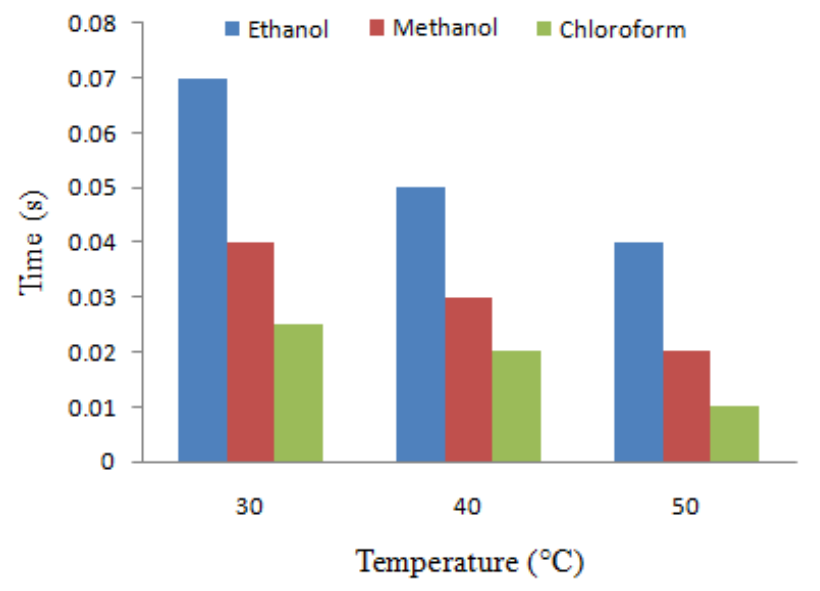

Fig.-4: Flow time at different temperatures.

The flow time for all liquids reduces with increase in temperature. Chloroform takes very less time to flow across channel at $50^{\circ} \mathrm{C}$ and maximum flow time at room temperature i.e. $30^{\circ} \mathrm{C}$. Ethanol takes maximum time to flow across the channel at $30^{\circ} \mathrm{C}$ and minimum flow time at $50^{\circ} \mathrm{C}$. Flow time for methanol is maximum at $30^{\circ} \mathrm{C}$ and minimum at $50^{\circ} \mathrm{C}$.

Velocity plot for all three liquids (Fig. 5) shows that the velocity of liquids flowing across the microchannel is temperature dependent The velocity of all liquids increases with increase in temperature. Chloroform is fastest among Methanol and Ethanol with maximum velocity at $50^{\circ} \mathrm{C}$ and minimum at room temperature i.e. $30^{\circ} \mathrm{C}$. Calculated temperature gradient of velocity at different temperatures is listed in Table III. It is observed that it decreases for Ethanol but increases for both Methanol and Chloroform. The investigations showed that the fluid can be identified automatically on the basis of microchannel flow rate and hence, the results may be very useful for developing microchannel based fluid sensors.

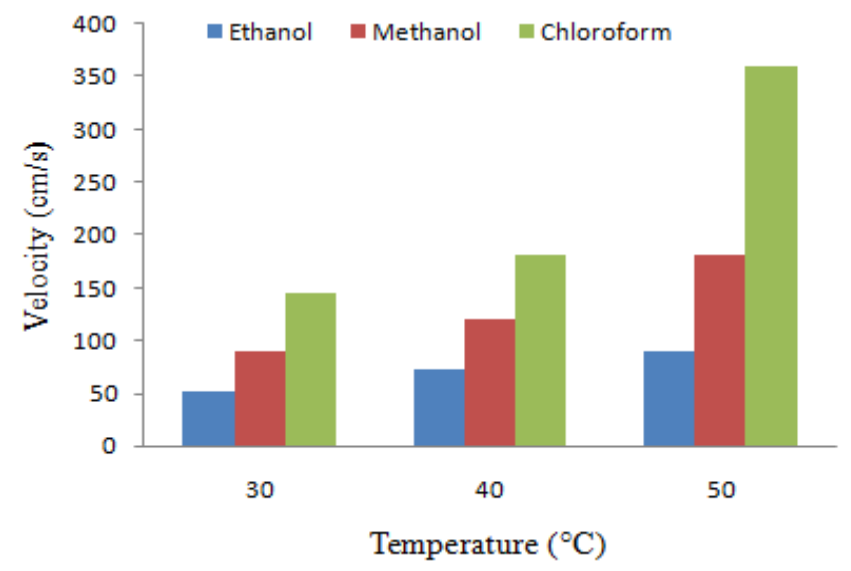

The temperature gradient of velocity at different temperatures is given below in Table 3 .

Table 3 Temperature Gradient of Velocity

\begin{tabular}{|l|c|c|}
\hline \multirow{2}{*}{ Microfluid } & \multicolumn{2}{|c|}{$\begin{array}{c}\text { Temperature gradient of velocity at } \\
\text { temperature }\left(\mathrm{cm} / \mathrm{s}^{0} \mathrm{C}\right)\end{array}$} \\
\cline { 2 - 3 } & $30^{0} \mathrm{C}$ & $40^{0} \mathrm{C}$ \\
\hline Ethanol & 2.1 & 1.8 \\
\hline Methanol & 3.0. & 6.0 \\
\hline Chloroform & 3.6 & 18 \\
\hline
\end{tabular}

\section{CONCLUSIONS}

Investigations were carried out to study the effect of temperature on the flow of Ethanol, Methanol, and Chloroform in a microchannel. It was observed that as the temperature increases to a higher value the flow pattern of Methanol and Ethanol becomes irregular as compared to regular flow shown by Chloroform. At higher temperature the speed of each fluid is more than that observed at room temperature. Thus fluid flow rate varies with changes in temperature. Higher the temperature faster is the speed of fluid flow across the microchannel. At high temperature the flow pattern transits from streamline to turbulent flow except for Chloroform. The investigations showed that the fluid can be identified automatically on the basis of flow analysis and hence, the results may be very useful for developing microchannel based fluid sensors.

\section{REFERENCES}

[1]. Radenovic, A., "Advanced Bioengineering Methods Laboratory Microfluidics Lab on Chip", http://tutorial6.com/m/microfluidics-lab-on-chip-e4141;

[1]. Unger, M. A., Chou, H. P., Thorsen, T., Scherer, A. \& Quake, S. R., "Monolithic Micro Fabricated Valves and Pumps by Multilayer Soft Lithography," in Science288, 113116 (2000). 14, 497-504, doi: 10.1016/S0958-1669(03)00113$7 ; 2000$

[3]. Paegel, B. M., Blazej, R. G. \& Mathies, R. A. Microfluidic Devices for DNA Sequencing: Sample Preparation and Electrophoresis analysis," in Current Opinion in Biotechnology 14, 42-50, doi: 10.1016/S09581669(02)00004-6; 2003.

[4]. Brackbill, J.U., Kothe, D.B., Zemach, C., "A Continuum Method for Modeling Surface Tension" in Journal of Computational Physics, Vol. 100; pp. 335-354; 1992.

[5]. Chakraborty, S. , "Dynamics of Capillary Flow of Blood into a Microfluidic channel, " in Lab on Chip, Vol. 5 part 4, pp. 421-30; 2005.

[6]. Schuyler Vowell, "Microfluidics: Effects of Surface Tension," in Physics 486 March 12, 2009.

Fig 5 Velocity at different temperatures 
[7]. R. M. Santos, M. Kawaji, "Effect of Contact Angle on Gas Slug Formation, Shape and Flow in a Microchannel Tjunction by Numerical Simulation," in. versita.com;

[8]. Shaw, J. M.; Gelorme, J. D.; Labianca, N. C.; Conley, W. E.; Holmes, S. J, "Negative Photoresists for Optical Lithography," in IBM Journal of Research and Development (1997), 41(1/2), 81-94;

[9]. Hiemenz, P. C.; Rajagopalan, R., "Principles of Colloid and Surface Chemistry," 1997, 3rd Ed., Marcel Dekker, Inc.; 1997

[10]. K. Grundke, "Wetting, Spreading and Penetration" in Handbook of Applied Surface and Colloid Chemistry," Holmberg, K. Ed., John Wiley \& Sons Ltd; 2002

[11]. J, Gray ML, Schmidt MA., "Microfabrication in Biology and Medicine," in Ann. Rev. Biomed. Eng., Vol. 1(1); pp. 401-425; 1999

[12]. Betancourt T, Brannon-Peppas L., "Micro- and Nanofabrication Methods in Nanotechnological Medical and Pharmaceutical devices." in Int. J. Nanomedicine Vol. 1part 4; pp. 483-495; 2006

[13]. Voldman J, Gray ML, Schmidt MA., "Microfabrication in Biology and Medicine," in Ann. Rev. Biomed. Eng.; 1(1):401-425.doi:10.1146/annurev.bioeng.1.1.401;1999

[14]. Tyler Nathaniel Hinkle, "Blood Model Plasma Separation in a Microfabricated PDMS Device Exploiting Capillary Action Driving Forces," a Thesis in 2008.

[15]. Nuttawut Lewpiriya Wong and Chun Yang, "ACDielectrophoretic Characterization and Separation of Submicron and Micron particles using Sidewall Ag PDMS Electrodes" in Biomicrofluidics; Vol. 6(1), pp. 12807128079; 2012.

[16]. Hardt, Steffen; Schönfeld, Friedhelm, "Microfluidic Technologies for Miniaturized analysis systems," in Springer, Vol. XXI, pp.393-438, 2007.

[17]. Irandoust, S., Andersson, B., "Liquid Film in Taylor flow through a Capillary" in Industrial and Engineering Chemistry Research Vol. 28, pp. 1684-1688; 1989. 